\title{
Difficult patient, or something else? A review of personality disorders
}

\section{Specific behaviors or expressed thoughts may signal a need for screening. Take into account an individual's strengths and limitations when designing a Tx approach.}

\section{$>$ THE CASES}

Winston $\mathrm{S}^{*}$ is a 23-year-old man referred by a psychiatrist colleague for primary care. He works delivering papers in the early morning hours and spends his day alone in his apartment mainly eating frozen pizza. He has worked solitary jobs his entire life and says he prefers it that way. His answers to questions lack emotion. He doesn't seem to have any friends or regular contact with family. He follows the medical advice he receives but can't seem to get out of the house to exercise or socialize. His psychiatrist was treating him with a selective serotonin reuptake inhibitor for depression when he was referred.

Denise $L^{*}$ is a 37-year-old woman who transferred to your practice because she says the previous practice's office manager was disrespectful and the doctor did not listen to her. She has been "very appreciative" of you and your "well-run office." You have addressed her fibromyalgia and she has shared several personal details about her life. In the following weeks, you receive several phone calls and messages from her. At a follow-up visit, she asks questions about your family and seems agitated when you hesitate to answer. She questions whether you remember details of her history. She pushes, "Did you remember that, doctor?" She also mentions that your front desk staff seems rude to her.

Ruth $B^{*}$ is an 82-year-old woman whose blood pressure measured in your office is $176 / 94 \mathrm{~mm} \mathrm{Hg}$. When you recommend starting a medication and getting blood tests, she responds with a litany of fearful questions. She seems immobilized by worries about treatment and equally so about the risks of nontreatment. You can't seem to get past the anxiety to decide on a satisfactory plan. She has to write everything down on a notepad and worries if she does not get every detail.

\section{OHOW WOULD YOU PROCEED WITH THESE PATIENTS?}

* This patient's name has been changed to protect his identity. The other 2 patients are an amalgam of patients for whom the authors have provided care.
Eric H. Berko, PhD;

Anthony J. Nedelman, PhD; Michael Raddock, MD

Case Western Reserve School of Medicine, Cleveland, OH (Drs. Berko and Raddock); MetroHealth Medical Center, Cleveland (Drs. Berko, Nedelman, and Raddock)

\section{$\Rightarrow$ mraddock@} metrohealth.org

The authors reported no potential conflict of interest relevant to this article. doi: $10.12788 / \mathrm{jfp} .0200$ 


\section{$>$}

The International Personality

Disorder

Examination

has a 15-minute

screening tool

to help identify

a personality

disorder,

regardless of

cluster.
A ccording to a survey of practicing primary care physicians, as many as $15 \%$ of patient encounters can be difficult. ${ }^{1}$ Demanding, intrusive, or angry patients who reject health care interventions are oftencited sources of these difficulties. ${ }^{2,3}$ While it is true that patient, physician, and environmental factors may contribute to challenging interactions, some patients who are "difficult" may actually have a personality disorder that requires a distinctive approach to care. Recognizing these patients can help empower physicians to provide compassionate and effective care, reduce team angst, and minimize burnout.

I What qualifies as a personality disorder? A personality disorder is an enduring pattern of inner experience and behavior that deviates markedly from the expectations of the individual's culture, is pervasive and inflexible, has an onset in adolescence or early adulthood, is unchanging over time, and leads to distress or impairment in social or occupational functioning. ${ }^{4}$ The prevalence of any personality disorder seems to have increased over the past decade from $9.1 \%{ }^{4}$ to $12.16 \% .^{5}$ The Diagnostic and Statistical Manual of Mental Disorders (DSM-5) classifies personality disorders in 3 clusters-A, B, and C (TABLE $)^{4}$-with prevalence rates at $7.23 \%, 5.53 \%$, and $6.7 \%$, respectively. ${ }^{5}$ The review below will focus on the distinct personality disorders exhibited by the patients described in the opening cases.

\section{A CLOSER LOOK AT THE CLUSTERS Cluster A disorders}

Paranoid, schizoid, and schizotypal disorders are part of this cluster. These patients exhibit odd or eccentric thinking and behavior. Individuals with schizoid personality disorder, for instance, usually lack relationships and lack the desire to acquire and maintain relationships. ${ }^{4}$ They often organize their lives to remain isolated and will choose occupations that require little social interaction. They sometimes view themselves as observers rather than participants in their own lives. ${ }^{6}$

\section{Cluster B disorders}

Dramatic, overly emotional, or unpredictable thinking and behavior are characteristic of individuals who have antisocial, borderline, histrionic, or narcissistic disorders. Patients with borderline personality disorder (BPD), for example, demonstrate a longstanding pattern of instability in affect, self-image, and relationships. ${ }^{4}$ Patients with BPD often display extreme interpersonal hypersensitivity and make frantic efforts to avoid real or imagined abandonment. Identity disturbance, feelings of emptiness, and efforts to avoid abandonment have all been associated with increased suicide risk. ${ }^{7}$

In a primary care setting, such a patient may display extremely strong reactions to minor disappointments. When the physician is unavailable for a last-minute appointment or to authorize an unscheduled medication refill or to receive an after-hours phone call, the patient may become irate. The physician, who previously was idealized by the patient as "the only person who understands me," is now devalued as "the worst doctor I've ever had."

\section{Cluster C disorders}

With these individuals, anxious or fearful thinking and behavior predominate. Avoidant, dependent, and obsessive-compulsive disorders are included in this cluster.

Dependent personality disorder (DPD) is characterized by a pervasive and extreme need to be taken care of. Submissive and clingy behavior and fear of separation are excessive. This patient may have difficulty making everyday decisions, being assertive, or expressing disagreement with others. ${ }^{4}$

Obsessive-compulsive personality disorder falls in this cluster and is typified by a pervasive preoccupation with orderliness, perfectionism, and control, at the price of flexibility and efficiency. This individual may be reluctant to get rid of sentimental objects, have rigid moral beliefs, and have significant difficulty working with others who do not follow their rules. ${ }^{4}$

\section{THESE CLUES MAY SUGGEST A PERSONALITY DISORDER}

If you find that encounters with a particular patient are growing increasingly difficult, consider whether the following behaviors, attitudes, and patterns of thinking are coming into play. If they are, you may want to consider 
using a screening tool, which we'll discuss in a moment.

\section{Clues to cluster A disorders}

- The patient has no peer relationships outside immediate family.

- The patient almost always chooses solitary activities for work and personal enjoyment.

\section{- Cluster B clues}

- Hypersensitivity to treatment disagreements or cancelled appointments are common (and likely experienced as rejection).

- Mood changes occur very quickly, even during a single visit.

- There is a history of many failed relationships with providers and others.

- The patient will describe an individual as both "wonderful" and "terrible" (ie, splitting) and may do so during the course of one visit.

- The patient may also split groups (eg, medical staff) by affective extremes (eg, adoration and hatred).

- The patient may hint at suicide or acts of self-harm. ${ }^{7}$

\section{- Cluster C clues}

- There is an excessive dependency on family, friends, or providers.

- Significant anxiety is experienced when the patient has to make an independent decision.

- There is a fear of relationship loss and resultant vulnerability to exploitation or abuse.

- Pervasive perfectionism makes treatment planning or course changes difficult.

- Anxiety and fear are unrelieved despite support and ample information.

\section{CONSIDER THESE SCREENING TOOLS}

Several screening tools for personality disorders can be used to follow up on your initial clinical impressions. We also highly recommend you consider concurrent screening for substance abuse, as addiction is a com-
TABLE

\section{Distinct personality disorders} within related clusters ${ }^{4}$

\begin{tabular}{l|l|l}
\hline Cluster A & Cluster B & Cluster C \\
\hline Paranoid & Antisocial & Avoidant \\
Schizoid & Borderline & Dependent \\
Schizotypal & Histrionic & Obsessive-compulsive \\
& Narcissistic & \\
\hline
\end{tabular}

mon comorbidity with personality disorders.

A sampling of screening tools. The Standardised Assessment of Personality Abbreviated Scale (SAPAS) ${ }^{9}$ is an 8-item measure that correlates well with disorders in clusters A and $\mathrm{C}$.

BPD (cluster B) has many brief scale options, including the McLean Screening Instrument for Borderline Personality Disorder (MSI-BPD). ${ }^{10}$ This 10-item questionnaire demonstrates sensitivity and specificity for BPD.

The International Personality Disorder Examination (IPDE) includes a 15-minute screening tool to help identify patients who may have any personality disorder, regardless of cluster. ${ }^{11}$

\section{IMPROVE PATIENT ENCOUNTERS WITH THESE TX PEARLS}

In the family medicine clinic, a collaborative primary care and behavioral health team can be extremely helpful in the diagnosis and management of patients with personality disorders. ${ }^{12}$ First-line treatment of these disorders is psychotherapy, whereas medications are mainly used for symptom management. See Black and colleagues' work for a thorough discussion on psychopharmacology considerations with personality disorders. ${ }^{13}$

The following tips can help you to improve your interactions with patients who have personality disorders.

\section{- Cluster A approaches}

- Recommend treatment that respects the patient's need for relative isolation. ${ }^{14}$

- Don't be personally offended by your patient's flat or disinterested affect or concrete thinking; don't
First-line treatment of personality disorders is psychotherapy; medications are mainly used for symptom management. 


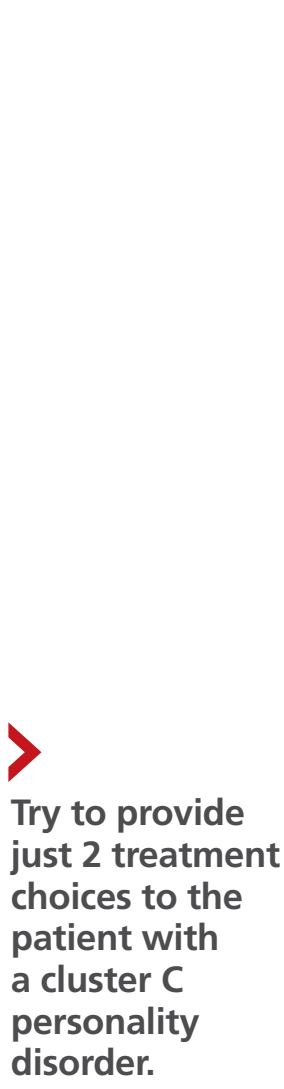

let it diminish the emotional support you provide. ${ }^{6}$

- Consult with a health psychologist (who has expertise in physical health conditions, brief treatments, and the medical system) to connect the patient with a longterm therapist. It is better to focus on fundamental changes, rather than employing brief behavioral techniques, for symptom relief. Patients with personality disorders tend to have better outcomes with long-term psychological care. ${ }^{15}$

\section{Cluster B approaches}

- Set boundaries-eg, specific time limits for visits-and keep them. ${ }^{8}$

- Schedule brief, more frequent, appointments to reduce perceived feelings of abandonment.

- Coordinate plans with the entire clinic team to avoid splitting and blaming. ${ }^{16}$

- Avoid providing patients with personal information, as it may provide fodder for splitting behavior. ${ }^{8}$

- Do not take things personally. Let patients "own" their own distress. These patients often take an emotional toll on the provider. ${ }^{16}$

- Engage the help of a health psychologist to reduce burnout and for more long-term continuity of care. A health psychologist who specializes in dialectical behavioral therapy to work on emotion regulation, distress tolerance, and interpersonal effectiveness would be ideal. ${ }^{17}$

\section{Cluster C approaches}

- Engage the help of family and other trusted individuals in supporting treatment plans. ${ }^{18,19}$

- Try to provide just 2 treatment choices to the patient and reinforce his or her responsibility to help make the decision collaboratively. This step is important since it is difficult to enhance autonomy in these patients. ${ }^{20}$

- Engage the help of a cognitive be- havioral therapist who can work on assertiveness and problem-solving skills. ${ }^{19}$

- Be empathetic with the patient and patiently build a trusting relationship, rather than "arguing" with the patient about each specific worry. ${ }^{20}$

- Make only one change at a time. Give small assignments to the patient, such as monitoring symptoms or reading up on their condition. These can help the patient feel more in control. ${ }^{21}$

- Present information in brief, clear terms. Avoid "grey areas" to reduce anxiety. ${ }^{21}$

- Engage a behavioral health provider to reduce rigid expectations and ideally increase feelings of self-esteem; this has been shown to predict better treatment outcomes. ${ }^{22}$

\section{CASES}

Mr. S displays cluster-A characteristics of schizoid personality disorder in addition to the depression he is being treated for. His physician was not put off by his flat affect and respected his limitations with social activities. Use of a stationary bike was recommended for exercise rather than walks outdoors. He also preferred phone calls to in-person encounters, so his follow-up visits were conducted by phone.

Ms. L exhibits cluster-B characteristics of BPD. You begin the tricky dance of setting limits, keeping communication clear, and not blaming yourself or others on your team for Ms. L's feelings. You schedule regular visits with explicit time limits and discuss with your entire team how to avoid splitting. You involve a psychologist, familiar with treating BPD, who helps the patient learn positive interpersonal coping skills.

Ms. B displays cluster-C characteristics of dependent and obsessive-compulsive personality disorders. At her follow-up visit, you provide a great deal of empathy and try not to argue her out of each worry that she brings up. You 
make one change at a time and enlist the help of her daughter in giving her pills at home and offering reassurance. You collaborate with a cognitive behavioral therapist who works on exposing her to moderately anxiety-provoking situations/decisions.
References

1. Hull SK, Broquet K. How to manage difficult patient encounters Fam Pract Manag. 2007;14:30-34.

2. Groves JE. Taking care of the hateful patient. $N$ Engl J Med. 1978;298: 883-887

3. O'Dowd TC. Five years of heartsink patients in primary care. $B M J$. 1988;297:528-530.

4. American Psychiatric Association. Diagnostic and Statistical Manual of Mental Disorders. 5th edition (DSM-5). American Psychiatric Publishing; 2013.

5. Volkert J, Gablonski TC, Rabung S. Prevalence of personality disorders in the general adult population in Western countries: systematic review and meta-analysis. Br J Psychiatry. 2018;213:709-715.

6. Esterberg ML, Goulding SM, Walker EF. Cluster A personality disorders: schizotypal, schizoid and paranoid personality disorders in childhood and adolescence. J Psychopathol Behav Assess. 2010;32:515-528.

7. Yen S, Peters JR, Nishar S, et al. Association of borderline personality disorder criteria with suicide attempts: findings from the collaborative longitudinal study of personality disorders over 10 years of follow-up. JAMA Psychiatry. 2021;78:187-194.

8. Dubovsky AN, Kiefer MM. Borderline personality disorder in the primary care setting. Med Clin North Am. 2014;98:1049-1064.

9. Hesse M, Moran P. (2010). Screening for personality disorder with the Standardised Assessment of Personality: Abbreviated Scale (SAPAS): further evidence of concurrent validity. BMC Psychiatry. 2010;10:10.

10. Zanarini MC, Vujanovic AA, Parachini EA, et al. A screening measure for BPD: the McLean screening instrument for borderline personality disorder (MSI-BPD). J Pers Disord. 2003;17:568-573.

11. Loranger AW, Sartorius N, Andreoli A, et al. The International Personality Disorder Examination. The World Health Organization/Alcohol, Drug Abuse, and Mental Health Administration international pilot study of personality disorders. Arch Gen Psychiatry. 1994;51:215-224.
12. Nelson KJ, Skodol A, Friedman M. Pharmacotherapy for personality disorders. UpToDate. Accessed April 22, 2021. www.uptodate.com/ contents/pharmacotherapy-for-personality-disorders

13. Black D, Paris J, Schulz C. Evidence-based integrated biopsychosocial treatment of borderline personality disorder. In: Muse M (ed). Cognitive Behavioral Psychopharmacology: the Clinical Practice of Evidence-Based Biopsychosocial Integration. Wiley; 2017:137-166.

14. Beck AT, Davis DD, Freeman A. Cognitive Therapy of Personality Disorders. 3rd ed. The Guilford Press; 2015.

15. Thylstrup B, Hesse M. "I am not complaining"-ambivalence construct in schizoid personality disorder. Am J Psychother. 2009;63:147-167.

16. Ricke AK, Lee MJ, Chambers JE. The difficult patient: borderline personality disorder in the obstetrical and gynecological patient Obstet Gynecol Surv. 2012;67:495-502.

17. Seow LLY, Page AC, Hooke GR. Severity of borderline personality disorder symptoms as a moderator of the association between the use of dialectical behaviour therapy skills and treatment outcomes. Psychother Res. 2020;30:920-933.

18. Nichols WC. Integrative marital and family treatment of dependent personality disorders. In: MacFarlane MM (Ed.) Family Treatment of Personality Disorders: Advances in Clinical Practice. Haworth Clinical Practice Press; 2004:173-204.

19. Disney KL. Dependent personality disorder: a critical review. Clin Psychol Rev. 2013;33:1184-1196.

20. Bender DS. The therapeutic alliance in the treatment of personality disorders. J Psychiatr Pract. 2005;11:73-87.

21. Ward RK. Assessment and management of personality disorders. Am Fam Physician. 2004;70:1505-1512.

22. Cummings JA, Hayes AM, Cardaciotto L, et al. The dynamics of self-esteem in cognitive therapy for avoidant and obsessivecompulsive personality disorders: an adaptive role of self-esteem variability? Cognit Ther Res. 2012;36:272-281.

This supplement was sponsored by Boehringer Ingelheim Pharmaceuticals, Inc. It was edited and peer reviewed by The Journal of Family Practice.

\section{Type 2 Diabetes 2021}

The management of type 2 diabetes (T2D) has changed substantially over the last several decades. Standard practices have moved away from the glucocentric approach of T2D management into an era in which the interplay among T2D, obesity, atherosclerotic cardiovascular disease, heart failure, and chronic kidney disease is increasingly recognized.

This supplement to The Journal of Family Practice brings together key updates in the field of T2D to help physicians care for patients who have not only T2D, but other interrelated diseases.

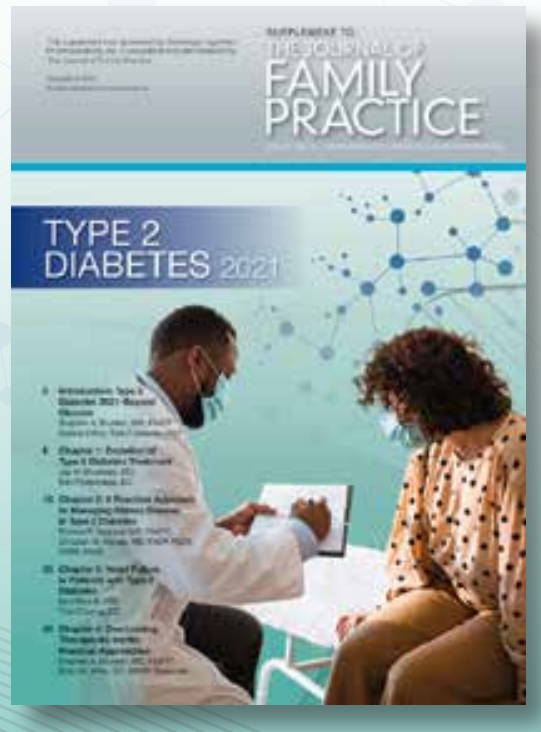

This supplement can be found at www.mdedge.com/T2D2021. 